\title{
Beliefs in being unlucky and deficits in executive functioning: An ERP study
}

Jaime Martin del Campo, Giorgio Fuggetta, John Maltby

There has been initial evidence to support the Dysexecutive Luck hypothesis, which proposes that beliefs in being unlucky are associated with deficits in executive functioning (Maltby et al., 2013). The present study tested the Dysexecutive Luck hypothesis by examining whether deficits in the early stage of top down attentional control led to an increase of neural activity in later stages of response related selection process among those who thought themselves to be unlucky. Individuals with these beliefs were compared to a control group using an Event-Related Potential (ERP) measure assessing underlying neural activity of semantic inhibition while completing a Stroop test. Results showed stronger main interference effects in the former group, via greater reaction times and a more negative distributed scalp late ERP component during incongruent trials in the time window of $450-780 \mathrm{~ms}$ post stimulus onset. Further, less efficient maintenance of task set among the former group was associated with greater late ERP response-related activation to compensate for the lack of top-down attentional control. These findings provide electrophysiological evidence to support the Dysexecutive Luck hypothesis. 
2 Running head: BELIEFS IN BEING UNLUCKY AND DEFICITS IN EXECUTIVE FUNCTIONING

3

4

5

6

7

8

9 College of Medicine, Biological Sciences and Psychology, University of Leicester, Lancaster Road,

10 Leicester, LE1 9HN, United Kingdom.jm420@le.ac.uk; jm148@le.ac.uk; gf43@leicester.ac.uk.

11

12

13

14 Corresponding Author: Jaime Martin del Campo, College of Medicine, Biological Sciences and

15 Psychology, University of Leicester, Lancaster Road, Leicester, LE1 9HN, United Kingdom. Tel: +44

16 (0) 116229 7198. Fax: +44 (0) 116229 7196. Email: jm420@le.ac.uk.

17

18

19
Beliefs in being unlucky and deficits in executive functioning: An ERP Study.

Jaime Martin del Campo

\author{
Giorgio Fuggetta
}

John Maltby 
20 There has been initial evidence to support the Dysexecutive Luck hypothesis, which proposes that

21 beliefs in being unlucky are associated with deficits in executive functioning (Maltby et al., 2013). The

22 present study tested the Dysexecutive Luck hypothesis by examining whether deficits in the early stage

23 of top-down attentional control led to an increase in neural activity in later stages of a response-related

24 selection process among those who thought themselves to be unlucky. Individuals believing themselves

25 to be unlucky were compared to a control group using an event-related potential (ERP), which

26 measured underlying neural activity of semantic inhibition, while they completed a Stroop test. Results

27 showed stronger main interference effects in the 'unlucky' group, via greater reaction times (RTs) and

28 a more negative distributed scalp late ERP component during incongruent trials in the time window of

$29450-780$ ms post-stimulus onset. Further, less efficient maintenance of task set among the former

30 group was associated with greater late ERP response-related activation to compensate for the lack of

31 top-down attentional control. These findings provide electrophysiological evidence to support the

32 dysexecutive luck hypothesis.

34 Keywords: Luck; Executive functions (EFs); Stroop; Event-related potential (ERP); Anterior cingulate 35 cortex (AAC) 


\section{Introduction}

38 Estimations of the prevalence of beliefs about luck in the general population are as follows: $50 \%$ of

39 people consider themselves lucky, 36\% consider themselves neither lucky nor unlucky, and the

40 remaining 14\% consider themselves to be unlucky (Wiseman, Harris \& Middleton, 1994). The most

41 prominent theory within the psychological literature on beliefs around luck is irrational belief theory,

42 which forms part of Rational Emotive Behavior Therapy (Ellis, 1994). According to this theory, beliefs

43 around luck reflect absolute beliefs about the world, where many aspects of life are attributable to

44 chance. Luck has an external, unpredictable, and uncontrollable influence upon the individual,

45 eventually forming the basis of emotional distress (Ellis, 1994). A more recent distinction has been

made between a perception of being lucky (belief in good luck) and the perception of being unlucky

47 (belief in bad luck) (Darke \& Freedman, 1997), with the latter being found to be associated with emotional distress, in terms of both hedonic and eudaimonic well-being (Maltby, Day, Gill, Colley \&

49 Wood, 2008).

Consequently there is literature that has focused on this distinction. One theory, the Dysexecutive

51 Luck hypothesis (Maltby, Day, Pinto, Hogan \& Wood, 2013), focuses on belief in being unlucky. The

52 Dysexecutive Luck hypothesis proposes that believing oneself unlucky is associated with lower levels

53 of executive functioning. There are two possible causal directions underpinning the Dysexecutive Luck

54 hypothesis. First, individuals' deficits in executive functioning may have a negative influence on their

55 ability to successfully achieve goals; as a result they label themselves unlucky. Second, individuals

56 believing themselves to be unlucky fail to engage the executive functions required for successful

57 completion of key goals. Maltby et al. (2013) showed some initial support for the Dysexecutive Luck

58 hypothesis. In that paper, self-report dysexecutive symptoms accounted for the unique variance in

59 beliefs in being unlucky after controlling for established correlates of luck beliefs (personality,

60 irrational beliefs, and self-efficacy). Experimental support for the Dysexecutive Luck hypothesis was 
61 demonstrated via significant positive correlations between individuals' beliefs of their being unlucky,

62 two (shifting and inhibition) of the three components (shifting, updating, and inhibition) of Miyake et

63 al.'s (2000) taxonomy of executive functions, and a significant negative correlation with decision-

64 making ability using somatic markers (Somatic Marker hypothesis; Damasio, Everitt \& Bishop, 1996).

The Dysexecutive Luck hypothesis places a key emphasis on the role of executive functioning.

Given the physiological basis of executive functioning (Jurado \& Rosselli, 2007), the consideration of whether the Dysexecutive Luck hypothesis occurs at a physiological level is important, if only to elucidate the psychological nature of the hypothesis. Currently, there is vicarious physiological evidence for the hypothesis arrived at using measures (e.g. Switch cost task, Stroop test, and IOWA Gambling Task) that are well-established proxies for physiological functioning. For example, taskswitching activates a common set of brain regions during diverse executive control operations, including medial prefrontal, superior and inferior parietal, medial parietal, and premotor cortices (Wager et al., 2004). Functional magnetic resonance imaging (fMRI) studies have shown that the IOWA Gambling Task is related to aspects of the prefrontal cortex ( $\mathrm{Li}$ et al., 2010) and the color-word Stroop test is related to activation in the frontal lobe of structures such as the dorsolateral prefrontal cortex (DLPFC) and the anterior cingulate cortex (ACC) (Spreen, Strauss \& Sherman, 2006; Lansbergen, van Hell \& Kenemans, 2007; Silton, Heller, Towers, Engels, Spielber \& Edgar, 2010). To begin this consideration, we addressed the Dysexecutive Luck hypothesis in terms of attentional control, because Maltby et al. (2013) found evidence for such hypothesis around key attentional processes. The cascade-of-control model proposes that during tasks that demand attention, the DLPFC takes a leading role in implementing top-down attentional control and later ACC activity is thought to be involved in resolving response-related attentional processes (Banich, 2009; Milham \& Banich, 2005; Silton et al., 2010). Previous studies assessing attentional control of the aging brain in 
84 healthy participants have provided evidence that during the color-word Stroop test, when early DLPFC

85 activity is relatively low, late ACC activity increases (Milham, Erickson, Banich, Kramer, Webb \&

86 Wszalek, 2002; Silton et al., 2010). The authors suggest that increased late response conflict is a

87 consequence of the reduced maintenance of a task set, which causes a need for increased ACC activity

88 in order to compensate for the lack of top-down attentional control required to maintain adequate task

89 performance (Milham et al., 2002; Silton et al., 2010).

The current study seeks to extend the Dysexecutive Luck hypothesis by providing initial

91 electrophysiological evidence supporting it. For this goal, scalp recorded event-related potentials

92 (ERPs) were recorded during the performance of a Stroop test. ERPs allow precise analysis of the time

93 course of neural events since they provide a real-time temporal resolution of neural processes by

94 reflecting event-locked electrical activity produced by neural ensembles (West \& Klein, 1999). This

95 electroencephalogram-derived technique was used thus to explore whether deficits in executive

96 functions in 'unlucky' individuals are associated with elongated (slow) reaction time, and whether

97 larger (more negative) late ERP waveforms underpinning response-related selection processes are

98 specifically associated with the interference effect (in order to compensate for the lack of top-down

99 control). The Stroop test was selected as the executive measure over other physiological assessments

100 (i.e. task switch) since it is widely regarded as a prototypical inhibition task (a main function of

101 attention control) (Miyake et al., 2000), a paradigmatic measure of selective attention (Carter, Mintum

$102 \&$ Cohen, 1995), and a well-established proxy for physiological functioning. Its main effect (i.e. Stroop

103 effect) is widely used in clinical practice and is the most extensively studied phenomena in

104 experimental psychology (MacLeod, 1991). Most importantly, the Stroop test was utilized to test the

105 Dysexecutive hypothesis for the first time in the study on which this study is based (Maltby et al.,

106 2013) and aims to further develop. 


\section{Method}

109

110

111

112

113

114

115

116

\subsection{Participants}

Initially, 217 undergraduate students (180 females; mean age $=20.34$; $\mathrm{SD}=2.1$ ) completed the 6-item Beliefs in Being Unlucky subscale from the Beliefs Around Luck scale (Darke \& Freedman, 1997; Maltby et al., 2008). Responses are scored on a 5-point scale ( $1=$ "Strongly Disagree" to 5="Strongly Agree"). Internal reliability estimates for this subscale are $\alpha>.85$ and the measure demonstrates validity via acceptable correlations between both peer and family ratings and predicted correlates of beliefs in being unlucky (Maltby et al., 2008). Participants were recruited from a university experiment participation scheme. The study was advertised and completed online via an electronic survey system.

Twenty-five students were selected from the initial group, all of whom had the highest (item mean $>3.4$ ) or lowest scores (item mean $<1.8$ ) on the Beliefs in Being Unlucky subscale. Five respondents' data was discarded and not considered in the statistical analysis due to either excessive eye-movement artifacts or inconsistent scores in a re-test of their beliefs around luck. Thus 20 students $(18$ women; mean age $=22.7 ; \mathrm{SD}=2.81)$ were selected for the final study. Two final experimental groups were formed: the 10 participants with the highest scores on the Beliefs in Being Unlucky subscale ('unlucky' group; mean $=4.63 ; \mathrm{SD}=.37$ ) and the 10 with the lowest scores on the Beliefs in Being Unlucky subscale (Control group; mean $=1.43 ; \mathrm{SD}=.47$ ). All participants had normal or corrected-to-normal vision, were unaware of the main purposes of the study, and had no history of any mental or neurological disorders. All participants, except one, were right-handed as assessed by the Edinburgh Handedness Inventory (Oldfield, 1971).

To establish further validity for the experimental groups we administered measures of dysexecutive symptoms (Wilson, Alderman, Burgess, Emslie \& Evans, 1996), neuroticism (Gosling et al., 2003) and self-efficacy (Chen, Gully \& Eden, 2001). This was to profile both experimental groups 
131 in terms of those characteristics found within the wider luck literature, namely that the belief in being

132 unlucky is positively associated with neuroticism and dysexecutive symptoms, and negatively

133 associated with self-efficacy (Maltby et al., 2008; 2013). A series of Mann-Whitney U comparisons

134 demonstrated that 'unlucky' individuals scored significantly higher on dysexecutive symptoms $(U=$

$1353.88 p<.001)$, neuroticism $(U=18.80, p=.016)$, and significantly lower on self-efficacy $(U=4.00, p$

$136<.001)$ than the control group.

Participants were paid 12.00 GBP for this study. The University of Leicester granted Ethical

138 approval to carry out the study within its facilities (Ethical Application Ref: jm420-c5a3d).

139

140 2.2. Experimental Measures.

141 The aim of the experimental measures was to record event-related potentials (ERPs) during a manual

142 color-word Stroop test. The construction of the current Stroop design was based on previous Stroop

143 ERP investigations (West \& Alain, 1999; Badzakova-Trajkov, Barnett \& Waldie, 2009) but varied on

144 two significant aspects.

The first modification was the addition of a neutral condition to the paradigm in order to fully match the stimuli conditions which now each respectively and separately holds a baseline comparison

147 (two experimental conditions and two control conditions), allowing more flexibility and a more 148 adequate statistical analysis. These two control conditions were required to be also balanced to the 149 main conditions in perceptual difficulty/conflict, so that they correspondingly represent either one 150 perceptive congruency or one perceptive incongruence only between them (See Table 1). Therefore, 151 control incongruent trials provided one cognitive incompatibility: a grapheme-length mismatch; and 152 control congruent trials delivered also a single cognitive compatibility: a grapheme-length match; 153 whereas the incongruent and congruent conditions respectively possess either two incompatibilities 
154 (one grapheme-length and one semantic content mismatch) or two compatibilities (one grapheme-

155 length and one semantic content match).

156 The second variance was the number of stimuli words matching for all conditions. The design

157 had the same number of control non-color words as the color words in the experimental conditions (4)

158 for a fully matched balanced design. Control non-color words were chosen carefully from the HAL

159 online database; were all stimuli matched in similar HAL frequency, same number of syllabus and

160 same part of speech (all stimuli were adjectives/ nouns). Furthermore, two parallel versions of the

161 Stroop were (still fully-matched) created differing only in the control non-color words, with the

162 intention of avoiding any possible attentional bias due to a more distracting word.

163

164

165

166

167

168

169

170

171

172

173 Lexical Decision Task Behavioural Results, i.e. similar mean RTs (ms); (4) Grapheme-length, words

174 that are matched by the same number of letters to their respective color-word; and (5) Syllables, all of

175 the control words matched the number of syllables of their respective color-word. 
176

177

178

179

180

181

182

183

184

185

186

187

188

189

190

191

192

193

194

195

196

197

198

199

\subsection{Procedure}

Participants were instructed that it was highly important during the recording to remain still, avoiding as much body movement as possible, to keep their eyes fixated at the center of the screen, and to blink only when necessary and preferably between trials when stimuli disappeared.

Subjects sat $57 \mathrm{~cm}$ from a computer screen, and were restricted by a head and chin rest installed between the chair and the screen to minimize head movements. Stimuli were presented at a resolution of $1,024 \times 768$ pixels on a 21 -inch monitor with a vertical refresh rate of $100 \mathrm{~Hz}$. The task and EEG triggering was constructed and generated by E-Prime 2.0 software (Psychological Software Tools, Pittsburgh, PA), running on a PC Pentium IV desktop computer. Responses were recorded using a serial response box that featured a 0 millisecond debounce period, which allows for a high precision in answer recording. Each trial started with $1000 \mathrm{~ms}$ of a white fixation cross (“+”) which was presented over a black background at the center of the screen. This was replaced with the target stimulus item shown for $1000 \mathrm{~ms}$. A blank screen followed lasting $700 \mathrm{~ms}$. Written feedback with a duration of 300 ms appeared at the center of the screen after incorrect ('wrong') and missing ('missing') responses. The speed of response timeframe remained unaltered for all trials with a total duration for each trial being $3000 \mathrm{~ms}$.

Participants were required to indicate the color of the font (red, blue, yellow, or green) of the stimuli shown at the center of the screen. They did this by pressing one of the four corresponding colored buttons on a button box using the index and middle fingers of the right and left hands. All target stimuli were presented in a random order, though all were presented in the font Courier New, with a font size of 25 and a bold font style. At the beginning of the acquisition and practice phases, and before the start of each test block, a message appeared on the screen instructing the participants to press the space bar to begin the block of trials. After the space bar was pressed, the screen was blank for $2000 \mathrm{~ms}$. 
The total duration of each Stroop experimental session recording was 30 minutes, and consisted of 201 three sequential phases:

a) A color-to-key acquisition phase designed to establish a strong mapping between the stimulus colors and the correct response keys. This phase was presented in a single block of 96 trials

\subsection{ERP Recording and analysis}

221 Continuous EEG signals were recorded by a DC 32-channel amplifier (1-kHz sampling rate, $250 \mathrm{~Hz}$

high cut-off frequency; Brain Products Inc., Germany). The EEG activity was recorded via a

Waveguard elastic cap, containing 64 unshielded and sintered Ag-AgCl electrodes (CAP-ANTWG64; 
224 ANT, Netherlands), with an electrode layout according to the international 10-5 electrode system. The 225 following electrodes were used during the recording FP2, F3, FZ, F4, FC5, FC1, FCZ, FC2, FC6, C3, $226 \mathrm{CZ}, \mathrm{C} 4, \mathrm{CP} 5, \mathrm{CP} 1, \mathrm{CP} 2, \mathrm{CP} 6, \mathrm{P} 7, \mathrm{P} 3, \mathrm{PZ}, \mathrm{P} 4, \mathrm{P} 8, \mathrm{PO} 7, \mathrm{PO} 3, \mathrm{PO} 4, \mathrm{PO} 8, \mathrm{O} 1, \mathrm{OZ}$, and $\mathrm{O} 2$. The right227 earlobe electrode served as on-line reference. EEG waveforms were re-referenced off-line to the 228 average of the right- and the left-earlobe electrodes. Two electrodes placed in a bipolar montage at 229 approximately $1 \mathrm{~cm}$ from the outer canthus of each eye served to record the horizontal electrode positioned below the right eye and Fp2 referenced to the right earlobe. Electrode impedance was kept below $5 \mathrm{~K} \Omega$. EEGs were epoched from $200 \mathrm{~ms}$ pre-stimulus-onset to $1000 \mathrm{~ms}$ post-stimulusonset. Each EEG epoch was inspected off-line, and those with ocular artifacts (as indicated by HEOG activity exceeding $\pm 30 \mu \mathrm{V}$ and VEOG activity exceeding $\pm 80 \mu \mathrm{V}$ ) were excluded from statistical analyses. Only ERP data for trials with correct responses were analyzed, therefore artifacts from eye movements and excessive noise were marked bad and discarded after a selective individual trial review of each participant's data. To help remove slow and sustained shifts in voltage (from non-neural origin) during data acquisition and reduce high-frequency noise, averaged ERPs were filtered using $0.05 \mathrm{~Hz}$ high-pass, $30 \mathrm{~Hz}$ low-pass and $50 \mathrm{~Hz}$ notch filters. Mean amplitudes of ERP waveforms in the time window of $450-780 \mathrm{~ms}$ relative to a $200 \mathrm{~ms}$ pre-stimulus baseline were obtained for each subject under each of the four conditions. This late ERP time window was chosen based on visual inspection and relevant source-ERP color-word Stroop research. In particular, a recent study (Silton et al., 2010) supported a role only for late ACC activity (520-680 ms), which is related to later aspects of response selection, in differentiating Stroop conditions. ERP mean amplitudes were measured for a selected group of 4 electrodes in the frontocentral scalp region (Fz, FC1, FCz, FC2). This region was chosen because it was likely to reveal the 
247 brain processing associated with cognitive control in a situation requiring effective inhibition of

248 distracting task-irrelevant information (Badzakova-Trajkov, Barnett, Waldie, \& Kirk, 2009).

\section{2.5. Variable creation and statistical analyses}

250 A semiautomatic filtering operation of raw reaction times (RTs) data was carried out by E-prime 2.0 in order to remove extremely slow (retardations) and extremely fast (anticipations) responses. Consistent 252 with other studies in the area (e.g. Fuggetta, 2006), an absolute exclusion criterion excluded RTs less than $150 \mathrm{~ms}$ and greater than $3000 \mathrm{~ms}$. The magnitudes of a variety of effects were computed and analyzed for analysis. These effects were obtained after computing three different combinations of conditions and mean averages in the Stroop test, such as the facilitation effect (control congruent condition minus congruent condition), interference effect (incongruent condition minus control incongruent condition), and Stroop effect (incongruent condition minus congruent condition). Mean error (\%), and Stroop condition effects (ms) for the correct trials were only used in the reported analysis of variance (ANOVA). Three differential effects of the factor condition on the two groups of participants were quantified on the basis of ERP mean amplitudes from a particular region of interest (fronto-central area) using four electrodes' activity ( $\mathrm{Fz}, \mathrm{FC} 1, \mathrm{FCz}$, and $\mathrm{FC} 2)$ during the time window of $450-780 \mathrm{~ms}$ post-stimulus-onset and reading that were reported in the ANOVA. For each ANOVA, the sphericity assumption was assessed using Mauchly's test. Greennhouse-Geisser epsilon adjustments for non-sphericity were applied where appropriate. Post hoc paired t-test were Bonferronicorrected for multiple comparisons. For statistical testing, $p<.05$ was considered significant.

\section{Results}

The mean percentage of errors in the Stroop test was $2.572 \pm 0.574 \%$ with the mean percentage Stroop effect $\mathrm{M}=4.24(\mathrm{SD}=1.5)$. An ANOVA showed a significant effect for the conditions $\left(F_{(1.2,}\right.$ 
$27121.6)=7.24, p=.002, \mathrm{yp}^{2}=.29$ ), with post hoc comparisons showing a significant difference between 272 facilitation effect compared to the interference effect (.308\% vs. $3.167 \%, p=.033)$, and the Stroop 273 effect $(.308 \%$ vs. $4.242 \%, p=.037)$. This means that participants in both groups had significantly more 274 errors in the interference and Stroop conditions than in the facilitation condition (Stroop and 275 interference conditions represent the conflict). These effects are expected for each Stroop test. There 276 was no significant main effect for group $\left(F_{(1,18)}=1.24, p=.280\right)$, meaning both groups performed in a 277 similar fashion across the conditions.

278 In terms of the experimental groups' performance on the Stroop test, descriptive statistics 279 (mean, accuracy, and RTses) for the three Stroop condition effects in the two groups are shown in 280 Table 2. Mean RTs for the magnitudes of the three condition effects on the Stroop test for both groups 281 282 283 284 were $78.15 \pm 9.39 \mathrm{~ms}$. An ANOVA for the mean RTs showed a main effect of 'Group' $\left(F_{(1,18)}=6.30\right.$, $\left.p=.022, \mathrm{yp}^{2}=.26\right)$. There was also a significant main factor "condition effect" $\left(F_{(1.2,21.2)}=55.9, p<\right.$ $\left..001, \mathrm{yp}^{2}=.76\right)$. Furthermore, there was a two-way interaction "condition effect by group" with $F_{(1.2,}$ 21.2) $=5.3, p=.027 \mathrm{yp}^{2}=.23$. Post hoc comparisons showed a significant difference between the 'unlucky' and control group for interference effect (68 vs. $132 \mathrm{~ms}, p=.027$ ), and Stroop effect (90 vs. $163 \mathrm{~ms}, p=.017$ ), but not in the facilitation effect (6 vs. $9 \mathrm{~ms}, p=.766$ ). 
Figure 1 shows the grand average ERPs of the three main condition effects for both

296 experimental groups. The time window where the greatest ERP activity occurred was the late time

297 window, $450-780 \mathrm{~ms}$, which showed a statistically significant main effect of 'group', with $F_{(1,18)}=$

$2987.17, p=.022, \eta 2=.285$. There was an overall effects significant difference with decreased ERP

299 amplitudes for the control group compared with the 'unlucky' group (0.169 vs. -2.674 $\mu$ V). More

300 importantly, a significant three-way interaction 'Electrode * Condition effect * Group' was also found

$301\left(F_{(6,108)}=2.21, p=.028 \eta 2=.109\right)$. Post hoc comparisons of the significant three-way interaction

302 revealed a significant difference in the amplitude of the late ERPs between the 'unlucky' group and the

303 control group. Notably, there was a significant difference in the magnitude of interference effect for

304 electrodes Fz (0.202 vs. $-3.788 \mu \mathrm{V}), \mathrm{FC} 1(-0.335$ vs. $-3.638 \mu \mathrm{V}), \mathrm{FCZ}(-0.078$ vs. $-3.663 \mu \mathrm{V})$, and FC2

$305(-0.324$ vs. $-3.752 \mu \mathrm{V})$, respectively (the study's region of interest). Moreover, there was a significant

306 difference in the magnitude of the facilitation effect between individuals with no beliefs in being

307 unlucky and individuals with beliefs in being unlucky for electrode Fz (0.608 vs. $-2.951 \mu \mathrm{V})$ and FC1

$308(0.753$ vs. $-2.821 \mu \mathrm{V})$.

309

310

- Insert Figure 1 here -

311

312

313

- Insert Figure 2 here - 
319 The first finding, consistent with Maltby et al. (2013), was that 'unlucky' individuals performed poorly

320 on the Stroop test and experienced higher interference main effects when compared to the control

321 group. The interference effect differences between groups are of great importance since it has been

322 emphasized that the overall Stroop effect is not enough for an accurate conflict measure; it is necessary

323 to have a comparison to a baseline neutral condition. The interference effect, which measures an

324 interference or "cost" relative to a neutral condition, is the most reliable and robust component within

325 the Stroop test (Henik \& Salo 2004; Macleod, 1991). Electrophysiological results supported the role of

326 late negative ERPs' amplitude due to incongruent trials in differentiating 'unlucky' individuals from the

327 control group in the magnitude of the interference effect.

In the current study, as in previous ERP studies, we used a difference waveform (incongruent condition - control incongruent condition waveforms) in an attempt to isolate the processes specifically

330 associated with the interference effect (Badzakova-Trajkov et al., 2009; (Badzakova-Trajkov et al.,

331 2009; Markela-Lerenc, Ille, Kaiser, Fiedler, Mundt \& Wesibrod, 2004). Although the late time

332 window chosen in the current investigation (between 450 and $780 \mathrm{~ms}$ ) is an unusual epoch for Stroop-

333 related effects, we were particularly interested in assessing late stage response-related processing,

334 which we hypothesized would be enhanced in the group of 'unlucky' individuals to compensate for

335 their impairment in high-order attentional control processes. A recent study employed the source-

336 waveform ERP mediation analysis (Hayes, 2013) and found that only late ACC activity (520-680 ms)

337 was correlated with the interference effect, distinguishing the waveforms between

338 incongruent/congruent conditions (Silton et al., 2010). Thus the findings demonstrated, as did previous

339 studies, that late stage response selection processes are specifically associated with ACC function

340 (Milham et al., 2003; Silton et al., 2010). Furthermore, an ERP study on the color-word Stroop test

341 (Liotti et al., 2000) found a significant difference in the incongruent relative to the congruent trials on a 
342 left temporoparietal cortex scalp region during a late time window of $600-700 \mathrm{~ms}$, supporting the role 343 of late stage response selection processes in the interference effect.

344 Overall, both the indirect behavioral and direct electrophysiological significant interference 345 effects of the present study can be interpreted with Silton et al. study (2010), which integrated fMRI 346 and ERP data to identify the time course of regional brain activity associated with top-down attentional 347 control during the execution of a color-word Stroop test. Silton et al. demonstrated that the degree to 348 which ACC influenced Stroop performance depended on the level of earlier DLPFC activity. When 349 DLPFC activity levels were high, there was little ACC impact on Stroop performance. This suggests that when DLPFC provides sufficient attentional control, ACC has a smaller effect on overt performance. The finding that ACC activity was not critical for performance when DLPFC activity was high is also consistent with a prior study (Milham, Claus \& Cohen, 2003). A similar pattern of neurophysiological activity has been found in the current study in the individuals of the control group.

354 This group's minimal level of late ERP interference effect and adequate level of behavioral 355 performance are direct evidence for this interpretation.

Furthermore, Silton et al., (2010) demonstrated that in the case of low DLPFC activity, there was a relatively high late ACC activity, which affected Stroop performance with a response pattern that involved slow RT responses. These results were consistent with the idea that ACC was compensating

359 for the lack of top-down DLPFC control (Silton et al., 2010). The pattern of results for 'unlucky'

360 individuals in the current ERP study echoes these previous results; the individuals exhibited a 361 significantly increased response conflict with greater RTs and larger late negative response-related 362 ERPs during incongruent trials, compared to the control group.

The increased facilitation effect exhibited only in ERPs could be the result of an early, nonstrategic priming effect (word 'red' in red font), or a deficiency in the strategic allocation of attention that may result in word-reading errors (participants read the word rather than name the color). 
366 In terms of the interference effect, both the RTs and ERP results complement each other quite well,

367 given that it is the only one of the three main condition effects revealing significant differences

368 between groups. A global interpretation of these results suggests that 'unlucky' individuals had a slower

369 processing of the interference or "cost" relative to a baseline condition in the interference effect,

370 translating to slower RTs and greater ERP magnitudes in this condition effect when compared to the

371 control group. Furthermore, the control group had adequate attentional resources available to perform

372 the task and quickly resolved the 'conflict condition,' and did not manifest a strong interference effect

373 in the late ERPs. This suggests that the conflict resolutions of the Stroop test occurred earlier in the

374 control group than in the group of 'unlucky' individuals.

The present study is observational and cross-sectional in nature (since no conditions were

376 experimentally manipulated), therefore it is important to highlight that the findings cannot establish

377 that neural dysfunction in the executive network caused participants beliefs in being unlucky. Neither

378 confirm, at a highly specific localization level, which specific structures of the brain were responsible

379 for the different main effects found in this investigation [mainly due the limited number of channels

380 (24) used in the electrophysiological data recording]. However, these effects do show an association

381 between self-reported beliefs and neural dysfunction, and also suggest an anatomical basis. The

382 electrodes' region of interest in the current findings (fronto-central scalp) is in agreement with other

383 ERP investigations that reveal a consistent neuroanatomical basis, correlating the Stroop effect with

384 strong activation in the DLPFC and especially the ACC (e.g. Carter \& Van Veen, 2007; Botvinick et

385 al., 1999). Taken together, these studies strongly support a conflict-monitoring hypothesis (Bush et al.,

386 2000), whose main premise identifies the ACC as the structure of the brain responsible for signalling

387 the occurrence of conflicts in information processing, triggering compensatory adjustments in

388 cognitive control. In a Stroop test this event can be specifically identified in the semantic conflict

389 generated by the incongruent stimuli. 
With respect to future research related to the current thesis work, there is an opportunity to

391 further examine some of the findings presented. For instance, a possible study based on two pending

392 ideas would consist on first seeing if the electrophysiological lead supporting the dysexecutive luck

393 with belief in bad luck in UK can be replicated in another country, and/or the potential to extend the

394 number of luck factors employed in that study and work with all four beliefs around luck factors. This

395 would signal if there were cross-cultural potential to the earlier work and something that could be

396 pursued, as a continuation of this thesis work. Additionally, it would be also interesting to examine

397 how the dysexecutive hypothesis might behave when applied to other related and common beliefs, such 398 as hope.

399 In summary, the findings provide physiological data that supports the dysexecutive luck

400 hypothesis. They suggest that increased response conflict in the context of deficits executive functions

401 of 'unlucky' individuals have probably caused a need for increased late ACC activity, which in turn

402 translated to lengthened RTs and increased magnitude of late ERPs primarily involved in response-

403 related processes. This alteration of regional neural activity also supports the concept introduced in

404 previous studies that ACC was compensating for the lack of DLPFC attentional control in the attempt

405 to maintain adequate task performance.

406

407 
408

409

410

411

412

413

414

415

416

417

418

419

420

421

422

423

424

425

426

427

428

429

430

\section{References}

Badzakova-Trajkov, G., Barnett, K. J., Waldie, K. E., \& Kirk, I. J. (2009). An ERP Investigation of the Stroop test: The role of the cingulate in attentional allocation and conflict resolution. Brain Research, 1253, 139 -148.

Balota, D.A., Yap, M. J., Cortese, M. J., Hutchison, K. A., Kessler, B., Loftis, B., Neely, J. H., Nelson, D. L., Simpson, G. B., \& Treiman, R. (2007). The English Lexicon Project. Behavior Research Methods, 39(3), 445-459.

Banich, M. T. (2009). Executive Function: The Search for an Integrated Account. Current Directions in Psychological Science, 18, 89-94.

Botvinick, M., Nystrom, L. E., Fissell, K., Carter, C. S., \& Cohen, J. D. (1999). Conflict monitoring versus selection-for-action in anterior cingulate cortex. Nature, 402, 179-181.

Bush, G., Luu, P., \& Posner, M. I. (2000). Cognitive and emotional influences in anterior cingulate cortex. Trends in cognitive sciences, 4(6), 215-222.

Carter, C. S., \& Van Veen, V. (2007). Anterior cingulate cortex and conflict detection: an update of theory and data. Cognitive, Affective, \& Behavioral Neuroscience, 7, 367-379.

Chen, G., Gully, S. M., \& Eden, D. (2001). Validation of a new general self-efficacy scale. Organizational Research Methods, 4, 62-83.

Damasio, A. R., Everitt, B. J., \& Bishop, D. (1996). The somatic marker hypothesis and the possible functions of the prefrontal cortex [and discussion].Philosophical Transactions of the Royal Society of London. Series B: Biological Sciences, 351(1346), 1413-1420.

Darke, P. R., \& Freedman, J. L. (1997). The belief in good luck scale. Journal of Research in Personality, 31, 486-511.

Ellis, A. (1994). Reason and Emotion in Psychotherapy. NY: Birch Lane Press. 
431 Fuggetta, G. P. (2006). Impairment of executive functions in boys with attention deficit/hyperactivity 432 disorder. Child Neuropsychology, 12, 1-21.

433 Gosling, S. D., Rentfrow, P. J., \& Swann, W. B. Jr., (2003). Avery brief measure of the big five 434 personality domains. Journal of Research in Personality, 37, 504-528.

Henik, A., \& Salo, R. (2004). Schizophrenia and the Stroop effect. Behavioral and cognitive neuroscience reviews, 3, 42-59.

Jurado, M.B., \& Rosselli, M. (2007). The elusive nature of executive functions: a review of our current understanding. Neuropsychological Review, 17, 213-33.

Lansbergen, M. M., van Hell, E., \& Kenemans, J. L. (2007). Impulsivity and conflict in the Stroop test: An ERP study. Journal of Psychophysiology, 21, 33.

Li, X., Lu, Z., D’Argembeau, A., Ng, M., \& Bechara, A. (2010). The Iowa Gambling Task in fMRI images. Human Brain Mapping, 41, 410-423.

Liotti, M., Woldorff, M. G., Perez III, R., \& Mayberg, H. S. (2000). An ERP study of the temporal course of the Stroop color-word interference effect. Neuropsychologia, 38, 701-711.

Lund, K., \& Burgess, C. (1996). Producing high-dimensional semantic spaces from lexical cooccurrence. Behavior Research Methods, 28, 203-208.

MacLeod, C. M. (1991). Half a century of research on the Stroop effect: an integrative review. Psychological Bulletin, 109, 163.

Maltby, J., Day, L., Gill, P., Colley, A. \& Wood, A. M. (2008). Beliefs around luck: Confirming the empirical conceptualization of beliefs around luck and the development of the Darke and Freedman beliefs around luck scale. Personality and Individual Differences, 45, 655-660. 
454 Maltby, J., Day, L., Pinto, D.P. \& Hogan, R.A. \& Wood, A.M. (2013). Beliefs in being unlucky and 455 deficits in executive functioning. Consciousness and Cognition, 22, 137-147.

456 Markela-Lerenc, J., Ille, N., Kaiser, S., Fiedler, P., Mundt, C., \& Weisbrod, M. (2004). Prefrontal457 cingulate activation during executive control: which comes first? Brain Res Cognitive Brain Research, 18, 278-287.

Milham, M. P., Banich, M. T., Claus, E. D., \& Cohen, N. J. (2003). Practice-related effects demonstrate complementary roles of anterior cingulate and prefrontal cortices in attentional control. Neuroimage, 18, 483-493.

Milham, M. P., \& Banich, M. T. (2005). Anterior cingulate cortex: an fMRI analysis of conflict specificity and functional differentiation. Human Brain Mapping, 25, 328-335.

Milham, M. P., Erickson, K. I., Banich, M. T., Kramer, A. F., Webb, A., Wszalek, T., et al. (2002). Attentional control in the aging brain: insights from an fMRI study of the stroop task. Brain Cognitoon, 49, 277-296.

Miyake, A., Friedman, N.P., Emerson, M. J., Witzki, A. H., Howerter, A., \& Wager, T. D. (2000). The unity and diversity of executive functions and their contributions to complex "frontal lobe" tasks: A latent variable analysis. Cognitive Psychology, 41, 49-100.

Oldfield, R. C. (1971). The assessment and analysis of handedness: the Edinburgh inventory. Neuropsychologia, 9, 97-113.

Silton, R. L., Heller, W., Towers, D. N., Engels, A. S., Spielberg, J. M., Edgar, J. C., et al. (2010). The time course of activity in dorsolateral prefrontal cortex and anterior cingulate cortex during topdown attentional control. NeuroImage, 50(3), 1292-1302.

Spreen, O., Strauss, E., \& Sherman, E. M. S. (2006). A compendium of neuropsychological tests: administration, norms, and commentary (pp. 477-499). Oxford [Oxfordshire]: Oxford University Press. 
478 Wager, T. D., Jonides, J., \& Reading, S. (2004). Neuroimaging studies of shifting attention: a meta479 analysis. Neuroimage, 22(4), 1679-1693.

480 West, R., \& Alain, C. (1999). Event-related neural activity associated with the Stroop test. Cognitive $481 \quad$ Brain Research, 8, 157-164.

482 Wilson, B. A., Alderman, N., Burgess, P. W., Emslie, H., \& Evans, J. J. (1996). BADS: Behavioral $483 \quad$ Assessment of the Dysexecutive Syndrome.

484 Wiseman, R., Harris, P., \& Middleton, W. (1994). Luckiness and psi: An initial study. Journal of the 485 Society for Psychical Research, 60, 1-15.

486

487

488

489

490

491

492

493

494

495

496

497

498

499 Acknowledgements 
500 The CONACYT in Mexico (National Council of Science and Technology) fully sponsors the 501 postgraduate research degree under which this study is completed.

502

\section{Authorship}

504 J. Maltby devised the original approach and developed the study concept. G. Fuggetta and J. Martin del

505 Campo designed the experiment. Testing and data collection was performed by J. Maltby for the first

506 part of the study (Belief around Luck Scale screening), and G. Fuggetta and J. Martin del Campo for

507 the second part of the study (scales and EEG recording). J. Martin del Campo and G. Fuggetta

508 performed the data analysis and interpretation. J. Martin del Campo drafted the manuscript; G.

509 Fuggetta and J. Maltby provided critical revisions, contributing heavily to its writing. All authors

510 approved the final version of the paper for submission.

511

512

513 
1

Image of Grand Average ERPs

GRAND AVERAGE ERPS OF THE THREE MAIN CONDITION EFFECTS FOR BOTH STUDY GROUPS. NOTES: ALL THE EFFECTS WERE OBTAINED FROM THE COMBINATION OF FOUR FRONTOCENTRAL ELECTRODES, OR REGION OF INTEREST (FZ, FC1, FCZ, AND FC2) DURING THE TIME WINDOW OF 450 - 780 MS. THE GREATEST EFFECT WAS FOUND ON THE INTERFERENCE EFFECT (A). THE NEXT FIGURE (SEE FIGURE 2) SHOWS INDIVIDUAL ERP'S OF EACH OF THESE FOUR MAIN ELECTRODES. 
ROI ACC $\mu \mathrm{V}$ Interference effect

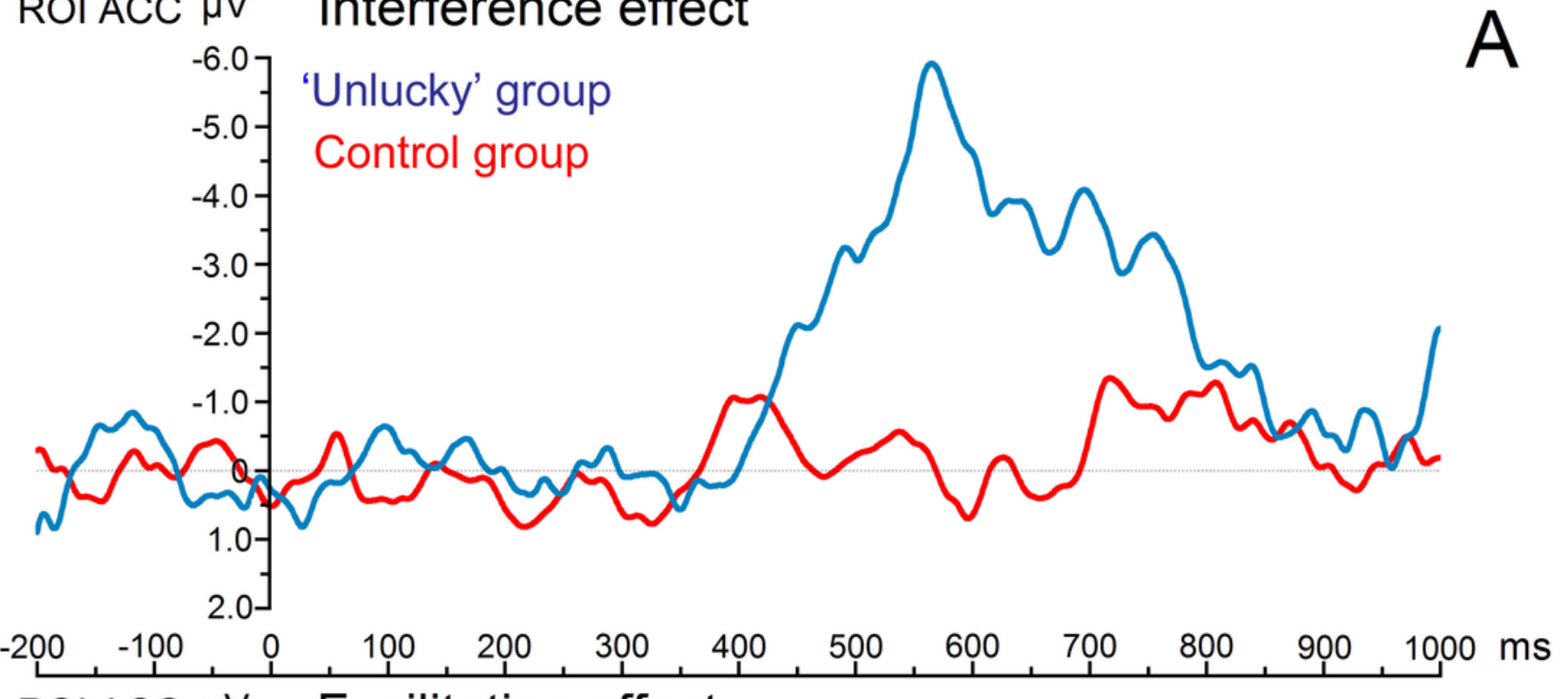
ROI ACC $\mu \mathrm{V}$ Facilitation effect

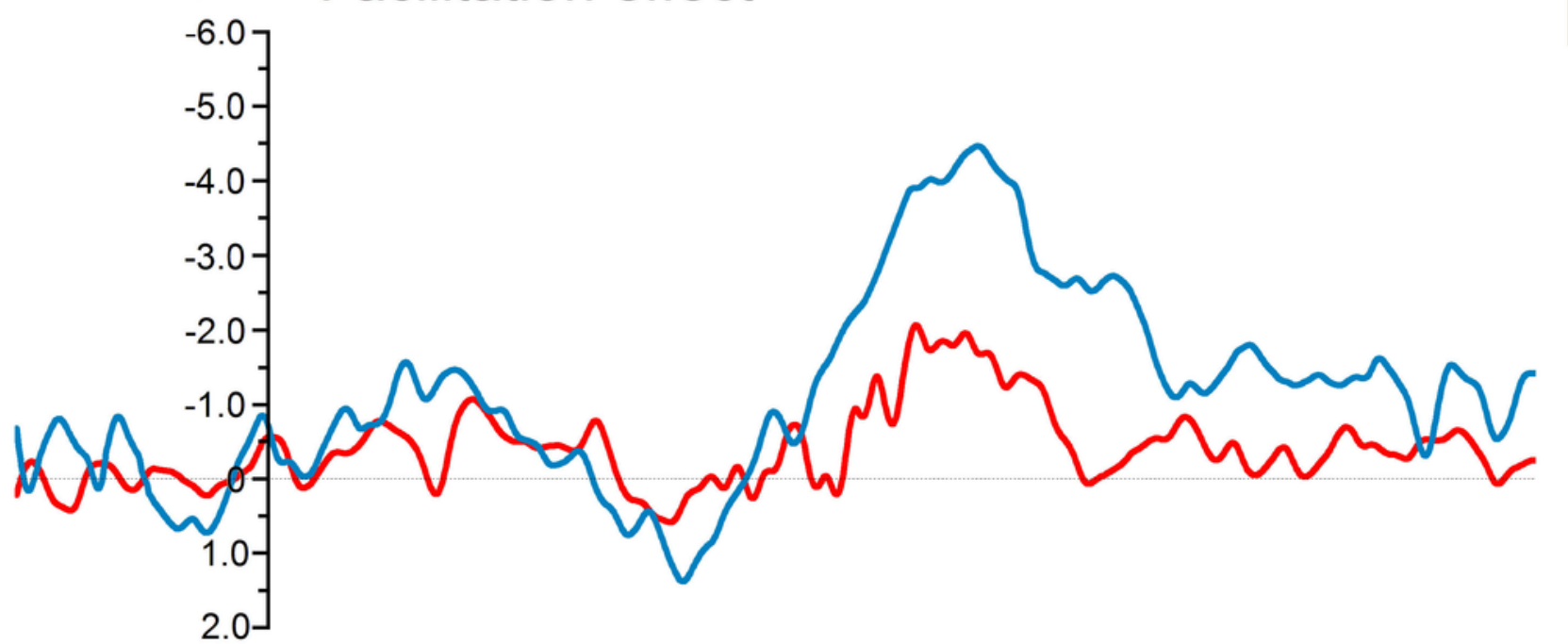

$-200,-100, \frac{1}{\perp}, 100,200,300,400,500,600,700,800,900,1000 \mathrm{~ms}$
ROI ACC $\mu \mathrm{V}$ Stroop effect

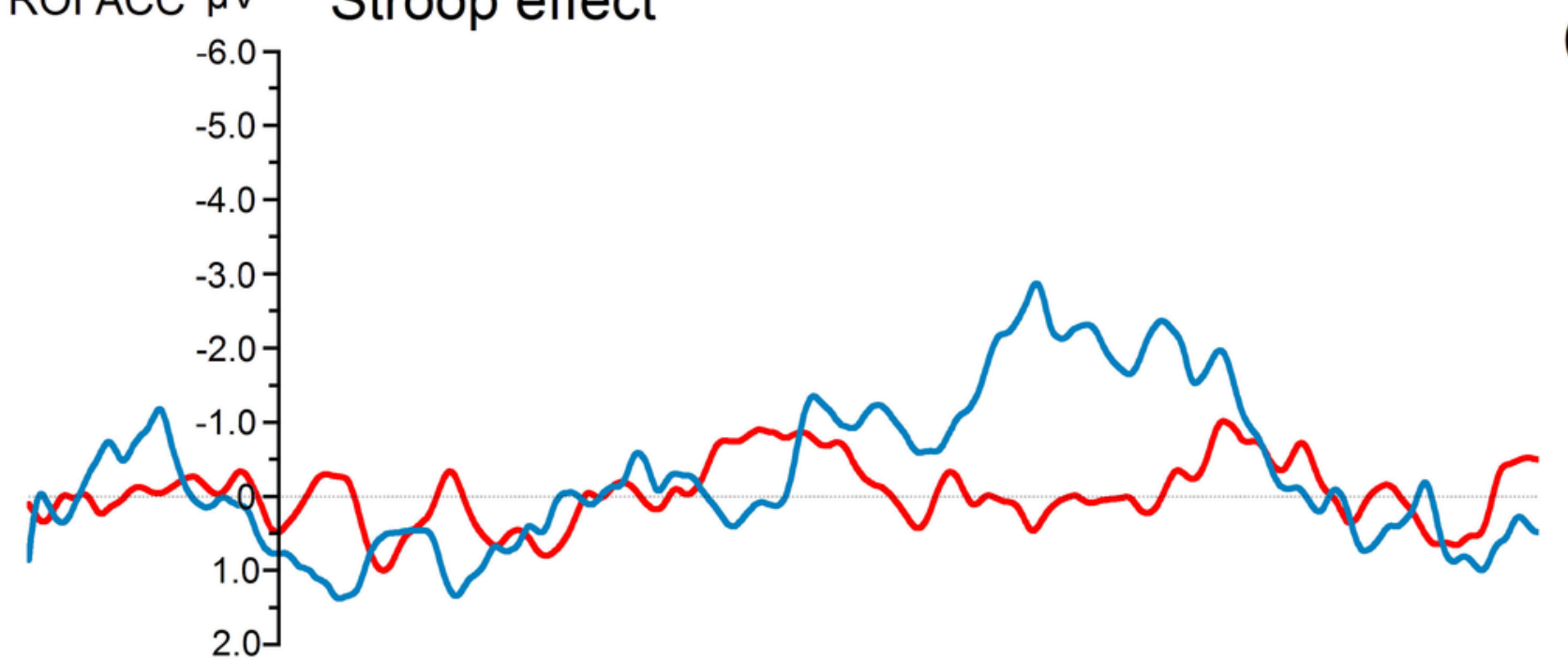

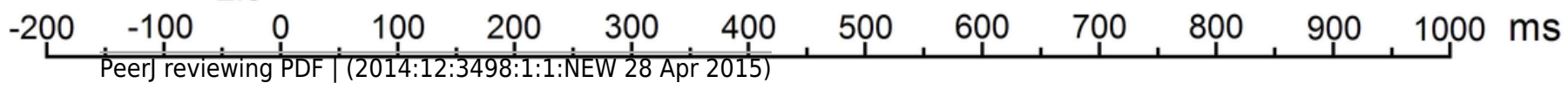


2

Image of Individual Electrodes ERPs

FOUR MAIN INDIVIDUAL ELECTRODES' ERP'S OF EACH STROOP TEST CONDITION (A1, A2, A3, AND A4) AND OF THE THREE MAIN CONDITION EFFECTS (B1, B2, B3, AND B4) FOR BOTH STUDY GROUPS. 
A1

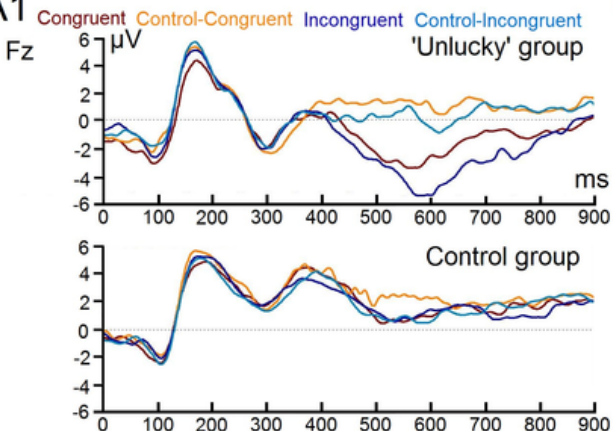

A2

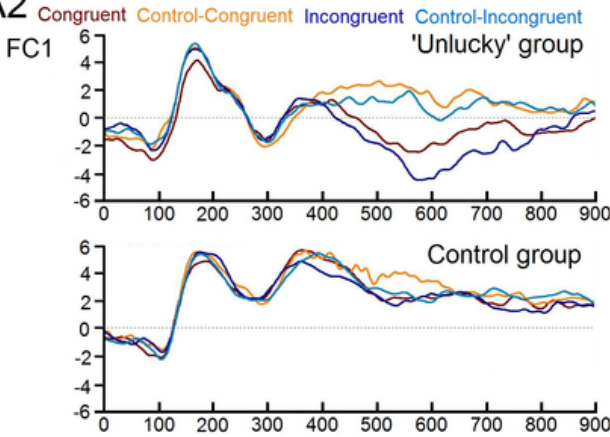

A3

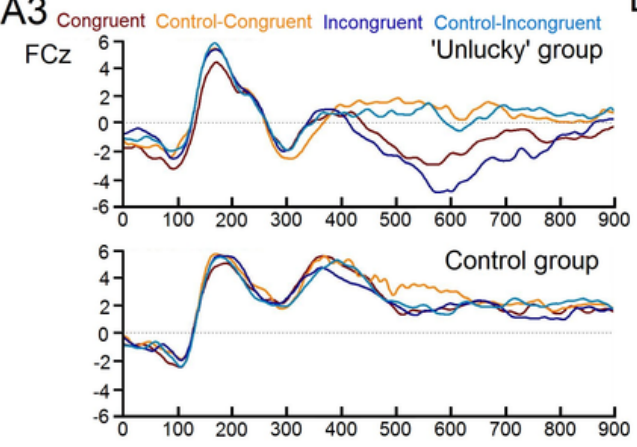

A4 congruent Control-Congruent Incongruent Control-Incongruent

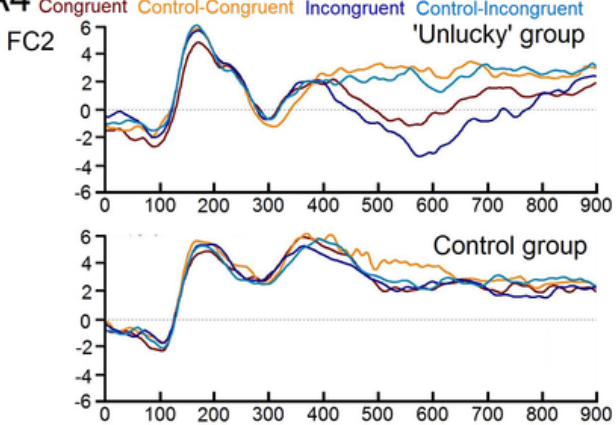

A5 congruent Control-Congruent Incongruent Control-Incongruent

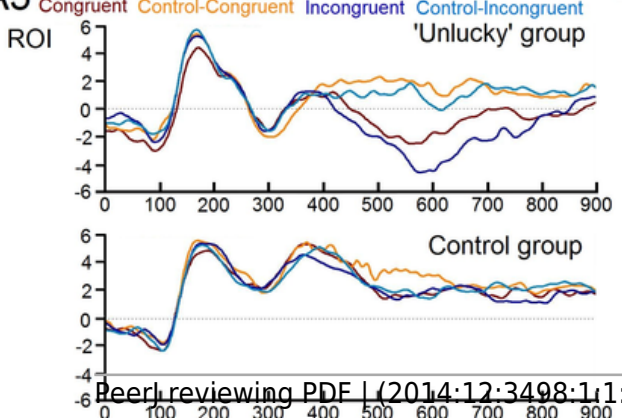

B1

Facilitation effect 'Unlucky' group Control group

FZ

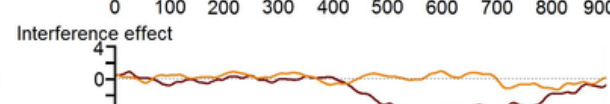

Stroop effect
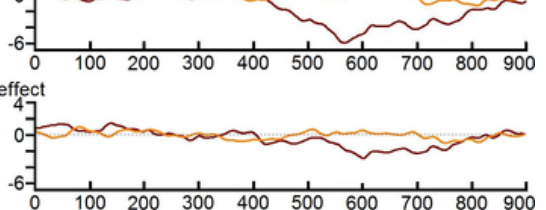

B2

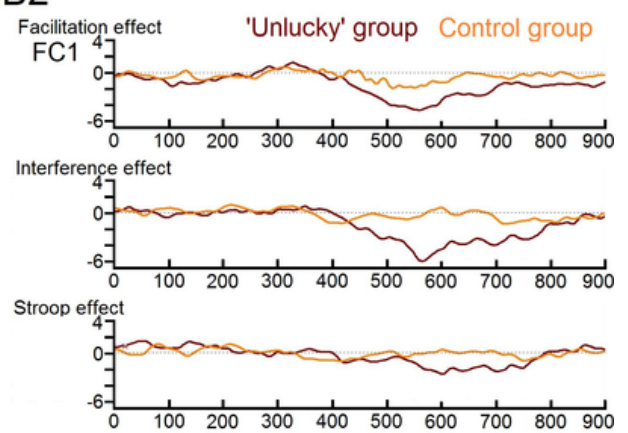

B3

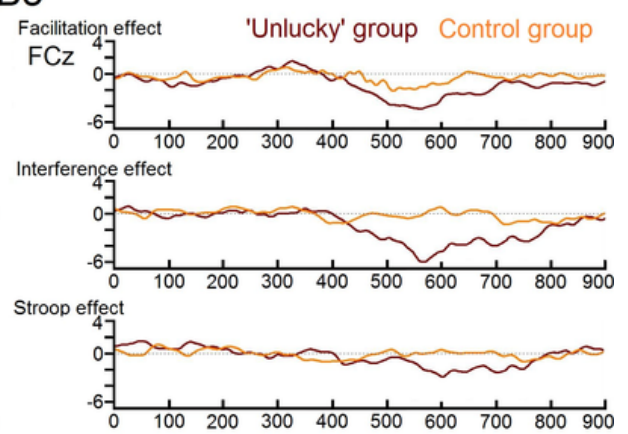

B4

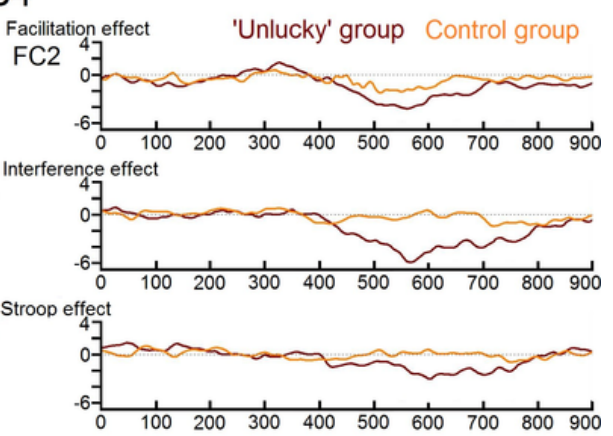

B5

Facilitation effect 'Unlucky' group Control group

$\mathrm{ROI}{ }^{4}$

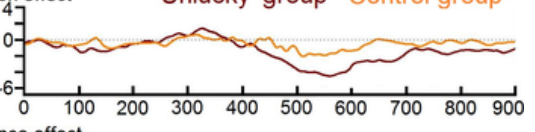

Interference effect

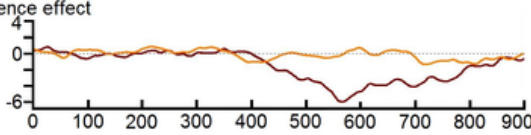

Stroop effect

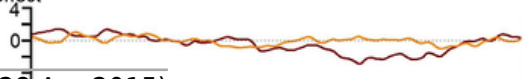


Table $\mathbf{1}$ (on next page)

Table containing examples of stimuli conditions used during the experimental phase of the Stroop task.

STIMULI USED DURING THE EXPERIMENTAL PHASE OF THE STROOP TASK. 
TABLE 1

3 STIMULI USED DURING THE EXPERIMENTAL PHASE OF THE STROOP TASK

\begin{tabular}{|c|c|c|}
\hline Stimulus & Word & Description \\
\hline \multirow[t]{2}{*}{ Condition } & Stimuli & \\
\hline & Examples & \\
\hline \multirow[t]{3}{*}{ Congruent } & 'red' (in & Word-color: matched \\
\hline & color red) & Word-color grapheme \\
\hline & & length: matched \\
\hline \multirow[t]{4}{*}{ Incongruent } & 'red' (in & Word-color: \\
\hline & color blue) & mismatched \\
\hline & & Word-color grapheme- \\
\hline & & length: mismatched \\
\hline \multirow{4}{*}{$\begin{array}{l}\text { Control } \\
\text { congruent }\end{array}$} & 'dog' (in & Word-color: neutral \\
\hline & color red), & Word-color grapheme- \\
\hline & 'jump' in & length: matched \\
\hline & color (blue) & \\
\hline Control & 'north' (in & Word-color: neutral \\
\hline \multirow[t]{3}{*}{ Incongruent } & color red), & Word-color grapheme- \\
\hline & 'deep' (in & length: mismatched \\
\hline & color yellow) & \\
\hline
\end{tabular}


Table 2 (on next page)

Table containing Mean Reaction Times and Mean Accuracy for both experimental groups

MEAN REACTION TIMES IN MILLISECONDS AND MEAN ACCURACY (\%) FOR BOTH EXPERIMENTAL GROUPS. 
2 TABLE 2

3 MEAN REACTION TIMES IN MILLISECONDS AND MEAN ACCURACY (\%) FOR BOTH EXPERIMENTAL 4 GROUPS.

\begin{tabular}{|c|c|c|c|}
\hline & \multicolumn{3}{|c|}{ MAIN CONDITION EFFECTS } \\
\hline & INTERFERENCE & FACILITATION & STROOP \\
\hline & (INCONGRUENT & (CONTROL & (INCONGRUENT \\
\hline & CONDITION - & CONGRUENT & CONDITION - \\
\hline & CONTROL & CONDITION - & CONGRUENT \\
\hline & INCONGRUENT & CONGRUENT & CONDITION) \\
\hline & CONDITION) & CONDITION) & \\
\hline \multicolumn{4}{|l|}{ CONTROL GROUP } \\
\hline REACTION TIME [MS] & $67.77(48.78)$ & $6.00(16.80)$ & $90.01(56.87)$ \\
\hline \multicolumn{4}{|l|}{ (SD) } \\
\hline ERRORS \% (SD) & $6.10(1.13)$ & $-1.33(0.88)$ & $5.5(1.48)$ \\
\hline \multicolumn{4}{|l|}{ 'UNLUCKY' GROUP } \\
\hline REACTION TIMES (MS) & $132.47(69.90)$ & $9.14(28.40)$ & $163.50(67.66)$ \\
\hline ERRORS \% & $3.92(4.55)$ & $.77(2.27)$ & $4.70(5.36)$ \\
\hline
\end{tabular}

5

6 